\title{
Water molecules in ultrashort intense laser fields
}

\author{
Simon Petretti ${ }^{a}$, Alejandro Saenz $^{\mathrm{a}, *}$, Alberto Castro $^{\mathrm{b}}$, Piero Decleva $^{\mathrm{c}}$ \\ ${ }^{a}$ AG Moderne Optik, Institut für Physik, Humboldt-Universität zu Berlin, Newtonstr. 15, D-12489 Berlin, Germany \\ ${ }^{\mathrm{b}}$ Institute for Biocomputation and Physics of Complex Systems (BIFI) and Zaragoza Center for Advanced Modeling (ZCAM), University of Zaragoza, E-50009 Zaragoza, Spain \\ ${ }^{\mathrm{c}}$ Dipartimento di Scienze Chimiche e Farmaceutiche and CNR-IOM Democritos and INSTM, Università di Trieste, Via L. Giorgieri 1, I-34127 Trieste, Italy
}

\section{A R T I C L E I N F O}

\section{Article history:}

Received 5 December 2011

In final form 18 January 2012

Available online 27 January 2012

\section{Keywords:}

Water

$\mathrm{H}_{2} \mathrm{O}$

Multiphoton ionization

Density-functional theory

Orientation dependence

Orbital imaging

Ultrashort laser pulses

Time-dependent Schrödinger equation

\begin{abstract}
A B S T R A C T
Ionization and excitation of water molecules in intense laser pulses are studied theoretically by solving the three-dimensional time-dependent electronic Schrödinger equation within the single-active-electron approximation. The possibility to image orbital densities by measurement of the orientation-dependent ionization of $\mathrm{H}_{2} \mathrm{O}$ in few-cycle, $800 \mathrm{~nm}$ linear-polarized laser pulses is investigated. While the highestoccupied molecular orbital $1 b_{1}$ is found to dominate the overall ionization behavior, contributions from the energetically lower lying $3 a_{1}$ orbital dominate the ionization yield in the nodal plane of the $1 b_{1}$ orbital. The ratio of the ionization yields of the two orbitals depends on the intensity. Furthermore, even for laser pulses as long as 8 cycles the orientation-dependent ion yield depends on the carrier-envelope phase. In the interpretation of the orientation-dependent ionization as an imaging tool these effects have to be considered.
\end{abstract}

(c) 2012 Elsevier B.V. All rights reserved.

\section{Introduction}

Recent efforts to achieve time-resolved imaging of chemical reactions or other structural changes with sub-femtosecond resolution possess a strong interdisciplinary character, since the goal is of interest for physics, chemistry, and biology. Pioneering experiments with near-infrared ultrashort laser pulses like the ones in $[1,2]$ in which structural information about the chemically most relevant valence electrons was extracted from the emitted high-harmonic radiation or electron spectra have been of great interest, since these approaches should intrinsically have the potential for providing also the required time resolution. However, in contrast to the initial assumptions, recent experiments have demonstrated that the molecular strong-field response depends, at least for some molecules, on more than one orbital [3-6]. While such multi-orbital effects clearly complicate simple imaging schemes, they can also be the source for even richer information that can be gained from such experiments. An example is the electron-hole dynamics in the lasergenerated ion that may be observed by analyzing the high-harmonic radiation [5]. Though very exciting by itself, this appears to make direct imaging of the valence electrons and their field-free dynamics during, e.g., a chemical reaction more complicated.

The proposed imaging schemes based on linear-polarized ultrashort near-infrared laser pulses may roughly be divided into two categories, rescattering-based schemes and direct imaging. The

\footnotetext{
* Corresponding author.

E-mail address: saenz@physik.hu-berlin.de (A. Saenz).
}

first category is based on the celebrated three-step model of strong-field physics in which (1) an electronic wavepacket leaves the molecule around the local maxima of the electric field by tunneling ionization, (2) this wavepacket is accelerated in the laser field and reverses its direction as the field direction changes, and (3) the electronic wavepacket may recollide with its parent ion. As a consequence of this recollision, the electronic wavepacket may partly scatter elastically (diffraction) or inelastically (leading to excitation or further fragmentation), or recombine by the emission of high-harmonic radiation. Clearly, all these processes should depend on the structure of the molecular ion and thus have the potential for revealing structural information. This includes both electronic structure as well as nuclear geometry. Corresponding reviews may be found in $[7,8]$.

\section{Direct imaging using orientation-dependent ionization}

While the rescattering-based imaging schemes are evidently based on the third step of the three-step model and consequently require that this model is at least a good approximation, the direct imaging schemes are based on the first, the ionization step. Measuring the ion yield as a function of the time-delay between two very short pulses has been demonstrated theoretically [9] and experimentally [10] to induce and image vibrational motion in the electronic ground state of neutral molecules. Simplified models like the strong-field approximation of the time-dependent Schrödinger equation suggest also the possibility to image the nuclear 
geometry of a molecule by comparing the energy-resolved electron spectra for different molecular orientations, but were immediately shown to fail using a full-dimensional ab initio treatment for $\mathrm{H}_{2}$ [11]. An alternative, rather straightforward approach to time-resolved direct imaging with intense near-infrared laser pulses is based on recording the dependence of the total ionization probability as a function of the relative orientation of the polarization axis of a linear polarized laser pulse with respect to the molecule. Since this corresponds to the fully integrated electron (or ion) spectrum, it is by far the most intense response signal, many orders of magnitude larger than recollision signals. This makes it experimentally very attractive. Clearly, it is an interesting question whether such an integral observable provides detailed enough information for imaging. In [12] it was demonstrated that the alignment-dependent ionization probability reflects the shape of the highest-occupied molecular orbital (HOMO) for both $\mathrm{N}_{2}$ and $\mathrm{O}_{2}$. However, the therein also considered example of $\mathrm{CO}_{2}$ turned out to be not as straightforward. A possible reason could be a coherent core trapping of the valence electron [13] which explains that only at lower intensity the imaging appears to work for $\mathrm{CO}_{2}$, in agreement with an experiment performed with lower laser intensity [14]. Alternative explanations for the shift of the peak positions have been given invoking either resonances [15] (not confirmed in [13]) or an interplay of coordinate and momentum properties of the HOMO [16].

Finally, it should be reminded that time-resolution is achieved in direct imaging schemes using pump-probe arrangements. Typically, the dynamics has anyhow to be triggered in some way. This may be achieved by two identical, time shifted laser pulses as in $[9,10]$ or by synchronizing another pump laser to the probe pulse. On the other hand, especially the high-harmonic based recollision imaging schemes possess an intrinsic time resolution that is, however, limited in total duration to about half a laser cycle and thus to about 1.6 fs for $800 \mathrm{~nm}$ laser light [17]. To follow dynamics on longer time scales, lasers with longer wavelengths have to be used, or a pump-probe scheme has to be applied as in the direct imaging schemes. Furthermore, the intrinsic time resolution can only be used for processes that are induced in the ionizing first step and thus not really adequate for the general purpose of imaging chemical reactions.

In order to image chemical reactions, it is, of course, important that concepts tested so far for linear molecules are also applicable to more general molecules. We have thus decided to investigate the direct imaging proposal based on the recording of the orientation-dependent ionization yield for a simple but very important non-linear molecule: water. Despite its natural importance due to its abundance in nature, there are some additional arguments for choosing this molecule in the present context. The three energetically highest-lying orbitals of $\mathrm{H}_{2} \mathrm{O}$ are known to very roughly correspond to the three $\mathrm{p}$ orbitals of the oxygen atom that are, however, differently modified by the $\mathrm{OH}$ bonds. All three orbitals are thus structurally relatively simple and a good test candidate for imaging schemes. For example, the energetically highest-lying occupied orbital, the $1 b_{1}$ HOMO, is almost identical to an oxygen $\mathrm{p}$ orbital. In contrast to true atomic p orbitals, the two hydrogen atoms in water lead, however, to a break of the isotropic symmetry and a deformation, especially of the so called $3 a_{1}$ HOMO- 1 . Furthermore, due to the symmetry reduction the three oxygen $\mathrm{p}$ orbitals loose their degeneracy and thus become (also energetically) distinguishable. As a consequence, it is possible to test the sensitivity of an imaging scheme. Note, it has to be mentioned that the field-free alignment of a dipolar molecule like $\mathrm{H}_{2} \mathrm{O}$ is experimentally a challenging task. However, orientation-resolved information for one-photon ionization was obtained in [18], but from a multiple coincidence measurement that allowed to reconstruct the orientation at the incident of ionization. Other approaches that avoid pre-alignment of the molecule are based on the use of circular polarized laser pulses as was applied to $\mathrm{H}_{2}$ in [19]. Clearly, in this case the theoretical description should consider circular and not linear polarization. Due to the increased numerical demands, this is, however, so far seldom the case (see, e.g., the theoretical analysis of the just mentioned $\mathrm{H}_{2}$ experiment in $\left.[19,11,20]\right)$.

\section{Previous studies of $\mathrm{H}_{2} \mathrm{O}$ in intense laser fields}

Ionization and harmonic generation of water molecules in intense fields have recently stirred some interest and were investigated both theoretically and experimentally. The case of relatively low intensities with supposedly negligible ionization was studied using time-dependent configuration interaction (restricted to single and double excitations) in [21] where polarizabilities and low harmonics were reported. Ionization of the HOMO of $\mathrm{H}_{2} \mathrm{O}$ in electric half-cycle pulses was studied in [22] within a hydrogenic-orbital approximation and thus an atomic model. Adopting a numerical implementation of time-dependent density functional theory (TD-DFT) based on Voronoi cells the ionization of water molecules in intense laser fields was studied in [23]. In that implementation only next-neighbor-cell interactions were considered. This approach will be in the following named TDVFD as in [23]. Considering two 2-dimensional cuts it was found that within the TDVFD approach the overall ionization is dominated by ionization from the HOMO, but at specific directions the HOMO-1 contribution can locally exceed the one from the HOMO. In [24] the ionization from the HOMO of water was calculated within the molecular Ammosov-Delone-Krainov (MO-ADK) approximation. At least along the considered two-dimensional cut the dependence of the ionization rate on the relative orientation between laser field and molecular plane as obtained by the MO-ADK model was shown to agree qualitatively, though not quantitatively, with the TDVFD results in [23].

The high-harmonic radiation of water molecules was recently investigated experimentally [25] and theoretically within a variant of the strong-field approximation in which the recombination dipole matrix element is related to single-photon ionization crosssections [26]. Already earlier, a theoretical investigation within the strong-field approximation was reported in [27]. In accordance with the in [23] predicted overall dominance of the HOMO ionization (first step of the three-step model), the theoretical investigation of isotope effects on the high-harmonic generation of water was restricted to the HOMO in [27]. It was concluded that practically no isotope effect is present and thus no nuclear motion is visible from the high-harmonic radiation, if the ratio of the highharmonic spectra from $\mathrm{H}_{2} \mathrm{O}$ and $\mathrm{D}_{2} \mathrm{O}$ is analyzed. The reason is the absence of (substantial) excitation of vibrational motion, if ionization from the water HOMO is considered. This is due to the fact that the potential surfaces of the neutral water molecule and the formed ion (in its electronic ground state) are very similar. The time-resolved imaging of nuclear motion using high-harmonics requires on the other hand vibrational excitation and thus different potential surfaces of the neutral and the ion $[9,28-30]$. Nevertheless, a recent mixed experimental and theoretical study [6] has shown a pronounced isotope effect in water harmonics. It is explained by high-harmonic generation from the HOMO-1 which, in contrast to the one from the HOMO, is accompanied by substantial vibrational excitation. As it becomes clear from the present study, the good visibility of the high-harmonic radiation from the HOMO-1 (relative to the one from the HOMO) is, within the terminology of the three-step model, also due to the second and third step, while ionization (in an isotropic ensemble) occurs dominantly from the HOMO, at least for not too high intensities. 
The main goal of the present work is, however, to provide quantitative predictions of the full three-dimensionally resolved dependence of the ionization yield of a water molecule on the relative orientation of the molecule with respect to the laser field axis. Based on these results the prospects of direct (time-resolved) imaging of orbital densities by recording the orientation or alignment dependent ionization probabilities are discussed.

\section{Method}

The full $a b$ initio treatment of the time-dependent response of molecules exposed to intense near-infrared laser fields is a nontrivial task. In fact, only very recently it has become possible to treat the two electrons of the simplest stable molecule, $\mathrm{H}_{2}$, exposed to intense ultrashort laser pulses (with $800 \mathrm{~nm}$ wavelength) for arbitrary orientations in full dimensionality [11]. Therefore, we have implemented a method that solves the time-dependent Schrödinger equation for in principle arbitrary molecules within the single-active-electron (SAE) approximation. The method was introduced in [31] where also the validity of the SAE approximation was demonstrated for $\mathrm{H}_{2}$. The extension to linear molecules with more than two electrons $\left(\mathrm{N}_{2}, \mathrm{O}_{2}\right.$, and $\left.\mathrm{CO}_{2}\right)$ followed in [13]. As it turned out, the standard frozen-core approximation is neither gauge invariant nor does it preserve the Pauli principle during the pulse, but it is possible to formulate a single-Slater-determinant approximation that does [32]. In the present work, almost all results were obtained with the latter approximation (named method $B$ in [13]). Since details of the approach can be found elsewhere $[6,13,31,32]$, it is only briefly described with an emphasis on the present implementation for general, non-linear molecules.

From the theoretical viewpoint the description of the ionization of non-linear molecules is challenging for two reasons. First, a proper description of the molecule has to account for its full multi-center character. Second, non-linear molecules belong to symmetry groups with no infinite symmetry element. Therefore, the use of symmetry does not lead to a dramatic reduction of the numerical efforts, since the Hamiltonian consists of a finite number of blocks and is by far not as sparse as for non-Abelian symmetries. The degree of sparseness determines on the other hand the efficiency of the time propagation. In the case of $\mathrm{H}_{2} \mathrm{O}$ with $C_{2 v}$ symmetry there are only four irreducible representations: $A_{1}, A_{2}, B_{1}$, and $B_{2}$. In view of this, compared to linear molecules, small reduction of the computational efforts it is evident that the present study demonstrates the ability of our recently developed approach $[13,31]$ to treat arbitrary molecules, because for the worst case, molecules with no symmetry $\left(C_{1}\right)$, the number of irreducible representations is only reduced from four to one.

In the first step of the calculation field-free Kohn-Sham orbitals are obtained using the LB94 exchange-correlation potential which has the correct asymptotic behavior [33]. The Kohn-Sham orbitals are described by a multi-centered $B$-spline basis [34]. This basis is composed of a set of (typically atom-centered) spheres in which a local basis is defined as the product of spherical harmonics and a radial part expressed in $B$ splines. These spheres must (presently) not overlap. An arbitrary number of such spheres centered at any position of $3 \mathrm{D}$ space is allowed. Molecular symmetry is accounted for by generating symmetry-adapted basis sets. A large central sphere that is usually positioned at the charge center of the molecule is added that defines an additional large $B$-spline basis. It overlaps with all atomic-centered spheres and provides an improved description of the chemical bonding and, especially, the molecular electronic continuum. The latter is obtained in a discretized form, the density of the discretized continuum being determined by the size of the central sphere. The concept is sketched for the present example of $\mathrm{H}_{2} \mathrm{O}$ in Fig. 1. Since the charge center lies very close to the oxygen nucleus, no local basis is defined for the latter, but

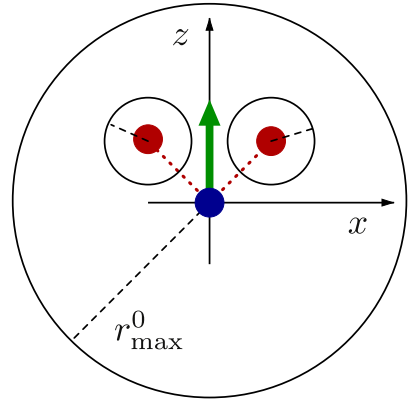

Fig. 1. Sketch (not to scale) of the spheres defining the local basis sets for $\mathrm{H}_{2} \mathrm{O}$. The origin is chosen to coincide with the oxygen atom (blue), while the two $\mathrm{H}$ atoms (red) and thus the molecule lie in the $x z$ plane. Two small spheres are centered around the hydrogen atoms, while one large sphere (with radius $r_{\text {max }}^{0}$ ) defines the main basis and discretization volume. The bold green arrow along the positive $z$ axis indicates the direction of the permanent dipole moment of water. (For interpretation of the references to colour in this figure legend, the reader is referred to the web version of this article.)

the center of the large sphere coincides with the oxygen nucleus and not with the charge center. It should be emphasized that the adopted multi-center basis is well suited to treat the atomic Coulomb singularities and the atomic cores of a molecule. For example, in the frozen-core SAE approach in [15] a fitted core potential had to be smoothened (by hand) in order to prevent numerical instabilities arising from the Coulomb singularities. Furthermore, much higher angular momenta are required for describing a multi-center molecule in a single-center basis. Also the Cartesian coordinates used in [35] are not optimal, as the slow quantitative convergence of that approach demonstrates.

In the present calculations, the radius of the large sphere is $r_{\max }^{0}=159.5$ a.u. and the maximum number of angular momenta is $l_{\max }=7$. The experimentally determined equilibrium geometry $\left(R_{\mathrm{OH}}=0.958 \AA, \Varangle \mathrm{HOH}=104.5^{\circ}\right.$ [37] $)$ is used and the water molecule lies in the $x z$ plane. The calculated eigenenergies of the KS orbitals occupied in the ground state are given in Table 1 and compared to experimental values taking the corresponding considerations in [38] into account.

The time-dependent wavefunction is expressed as a single Slater determinant; the initial state being the ground-state KohnSham Slater determinant. The one-electron wavefunctions that form the time-dependent Slater determinant are expanded in the basis of the occupied and virtual Kohn-Sham orbitals of the field-free Kohn-Sham molecular Hamiltonian. Thus the KohnSham orbitals form a convenient basis, but in principle any other complete single-particle basis could be used, like, e.g., the Hartree-Fock basis additionally used in [31]. It should be emphasized that the present approach is not TD-DFT. In contrast, the orbitals are propagated in the (frozen) ground-state Hamiltonian plus the external field. However, in contrast to standard SAE approaches (like method A in [13]) the interaction with the laser field modifies all orbitals during the laser pulse, not only the active one. As is shown in [32], the time-propagation of the Slater determinant can be reduced to an independent time propagation of the initially occupied orbitals. The ionization yield is defined as the population

Table 1

Calculated vertical ionization potentials $\left(I_{p}\right)$ for the occupied orbitals of water compared to experimental values.

\begin{tabular}{lll}
\hline Orbital & $I_{p}(\mathrm{eV})$ & \\
\cline { 2 - 3 } & Calculated & Experiment [36] \\
\hline $1 b_{1}$ & 13.15 & 12.61 \\
$3 a_{1}$ & 15.09 & 14.73 \\
$1 b_{2}$ & 18.69 & 18.55 \\
$2 a_{1}$ & 30.50 & 32.20 \\
$1 a_{1}$ & 535.16 & 539.70 \\
\hline
\end{tabular}


of all field-free continuum orbitals after the pulse. The total population is normalized to 1 , an ionization yield of, e.g., 0.1 corresponds to $10 \%$ ionization. As excitation we define the population of all field-free, initially unoccupied (virtual), but non-continuum orbitals after the pulse. The inclusion of orbitals with an energy lower than 20 a.u. led to about 6000 orbitals that were used in the calculations where the laser field is parallel to the $x, y$, or $z$ axis due to symmetry and dipole-selection rules, while about 12,000 orbitals were used for arbitrary orientations. The laser pulses were modeled using a $\cos ^{2}$ envelope for the vector potential,

$A(t)=A_{0} \cos ^{2}\left(\frac{\pi t}{T}\right) \sin (\omega t+\phi)$,

with total pulse duration $T$, laser frequency $\omega$, and carrier envelope phase $\phi$. Note that a pulse duration $T=8$ cycles corresponds to a full width half maximum of 2.9 (4) cycles with respect to intensity (electric field). The ionization yields are multiplied with a factor 2 , since all orbitals of $\mathrm{H}_{2} \mathrm{O}$ are doubly occupied (and non-degenerate). This procedure was shown to be appropriate for $\mathrm{H}_{2}$, except for very high ionization yields (above about $10-20 \%$ ) where a factor 1 should be used [31]. The calculations were performed in length gauge and within the interval $\varphi \in[0, \pi / 2]$ and $\vartheta \in[0, \pi]$, where $\varphi$ and $\vartheta$ represent the usual spherical coordinates. The full threedimensional representations are obtained by symmetry arguments.

\section{Results}

The shapes (iso-surfaces of identical position-space density) of the three energetically highest lying occupied molecular orbitals of $\mathrm{H}_{2} \mathrm{O}$ are visualized in the left panel of Fig. 2. Ordered by
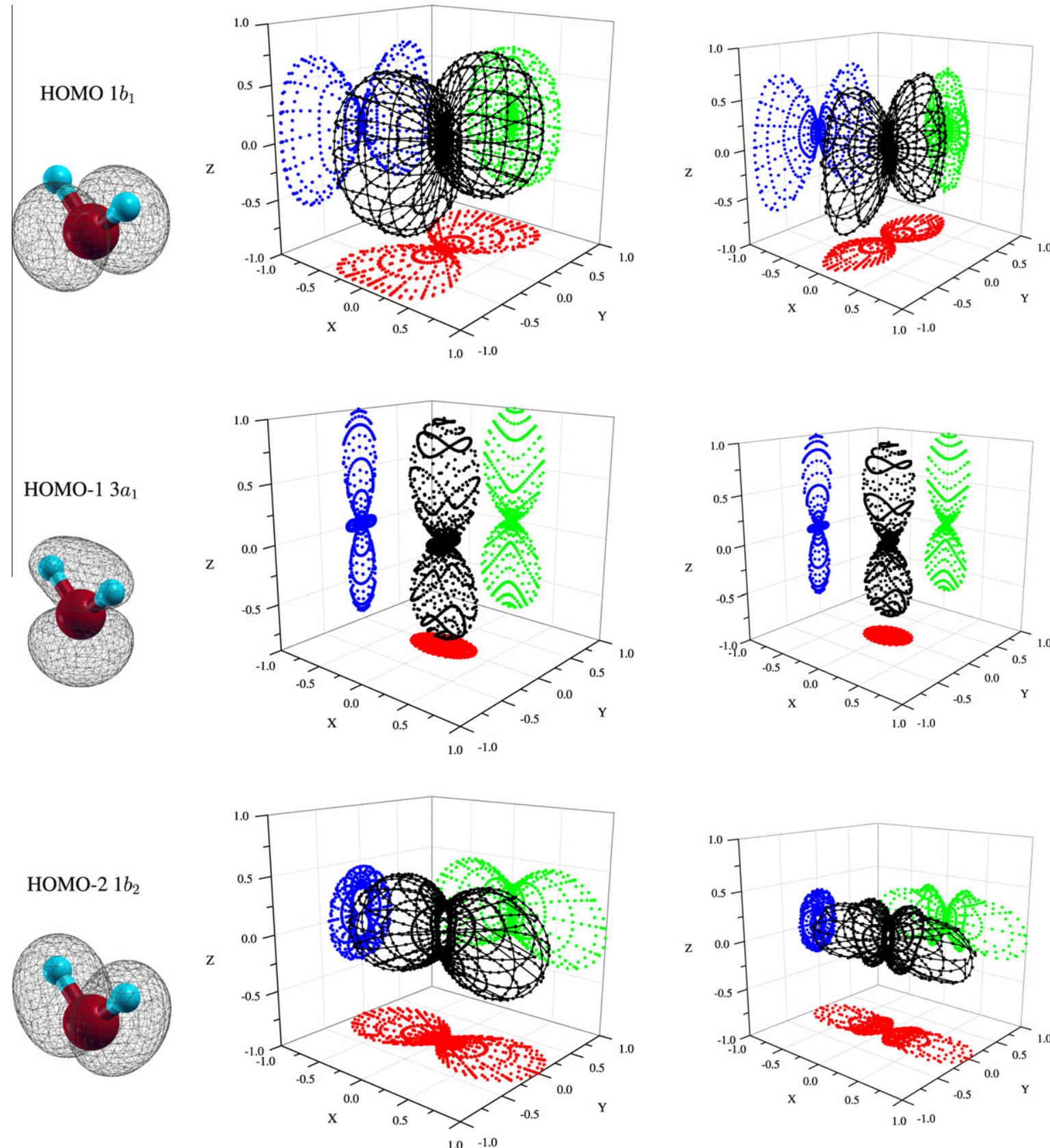

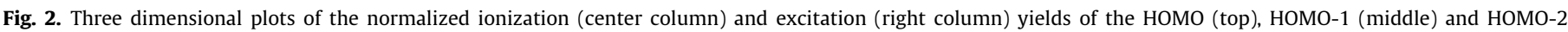

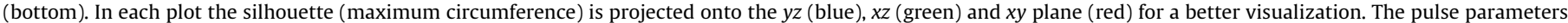

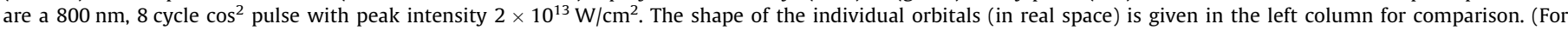
interpretation of the references to colour in this figure legend, the reader is referred to the web version of this article.) 


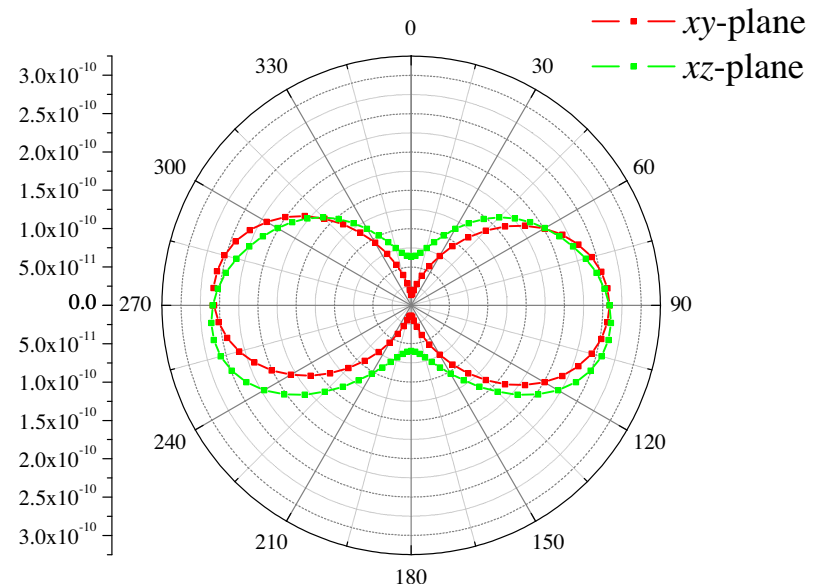

Fig. 3. Comparison of the cuts of the ionization yield of the HOMO-2 in $x y$ and $x z$ plane. The color coding agrees with the one in Fig. 2. (For interpretation of the references to colour in this figure legend, the reader is referred to the web version of this article.)

increasing electron binding energy, the orbitals are denoted as HOMO $\left(1 b_{1}\right)$, HOMO- $1\left(3 a_{1}\right)$, and HOMO-2 $\left(1 b_{2}\right)$. The response of $\mathrm{H}_{2} \mathrm{O}$ to an 8 -cycle $\cos ^{2}$ pulse (see Eq. (1)) with a central wavelength of $800 \mathrm{~nm}$, the laser peak intensity $2 \times 10^{13} \mathrm{~W} / \mathrm{cm}^{2}$, and $\phi=0$ is also shown in Fig. 2. The ionization yields (middle column) and excitation yields (right column) are normalized with respect to the maxima, i.e. the maximum yield in each plot is one. In agreement with the shapes of the orbitals the ionization yields show approximately p-orbital like structures that point along the directions of maximal extension of the orbitals. This is most evident for the HOMO that also itself is closest to an undistorted $\mathrm{p}$ orbital of an oxygen atom (the $\mathrm{p}_{y}$ in our chosen coordinate system).

The HOMO-2 is also rather similar to an oxygen p orbital (the $\mathrm{p}_{x}$ ), but the two lobes are distorted in the direction of the hydrogen atoms (within the $x z$ plane). The ionization yield of the HOMO-2 is not rotationally symmetric around the $y$ axis, but shows a similar distortion in the $x z$ plane as the orbital itself. This can be seen even more clearly in the corresponding cuts shown in Fig. 3 in which the absolute values of the ionization yield as a function of the orientation of the molecule with respect to the field axis is shown. However, the distortion is opposite to the one of the orbital, since it points toward the negative $z$ axis, while the hydrogen atoms point toward positive $z$. In fact, one would not expect any asymmetry of this kind in in the strong-field response to a linear polarized laser field, since the electric field vector points periodically "up" and "down". Therefore, the total ionization probability should be inversion symmetric. The found asymmetry is a consequence of the shortness of the adopted laser pulse, though 8 cycles is not ultrashort. As a check, a calculation with a different carrier envelope phase $\phi=\pi$ (instead of 0 ) was performed. As expected, the ionization yields are a mirror image of the ones shown in Figs. 2 and 3, i.e. the lobes of the HOMO-2 point for $\phi=\pi$ toward positive $z$. The strong phase dependence found here even for a pulse as long as 8 cycles is very likely to be a consequence of the strong dipole moment of (oriented) water molecules. Thus, a very pronounced carrier envelope effect should occur for even shorter laser pulses. Since the dipole vector of the molecule is oriented along the $z$ direction and the orbital density possesses mirror symmetry with respect to the $y z$ and $x z$ planes, the asymmetry occurs in the $x z$, but not in the $x y$ plane. While the strong phase effect is interesting by itself and provides in sufficiently short pulses even information on the absolute orientation of a molecule and not only its alignment, it is problematic from the viewpoint of orbital imaging. In the case of the HOMO-2 of water it could appear as the correct
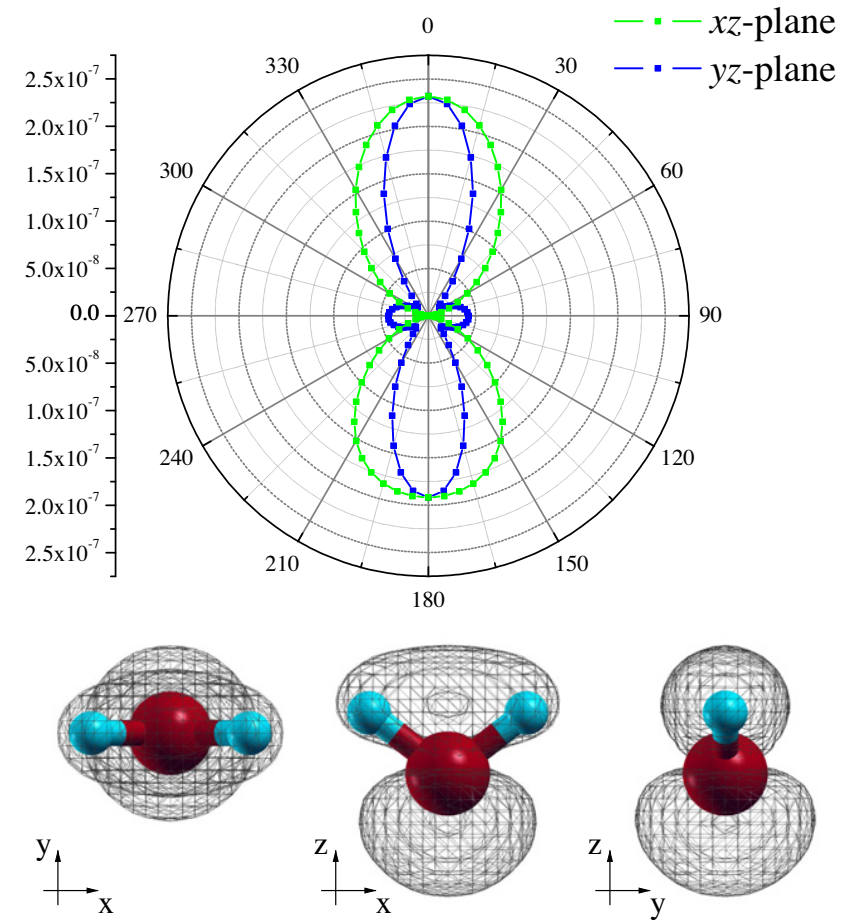

Fig. 4. Shown are cuts of the ionization yield of the HOMO-1 in the $x z$ and $y z$ plane. The color coding agrees with the one in Fig. 2. Below, the shapes of the HOMO-1 projected on the $x y, x z$ and $y z$ plane are represented. (For interpretation of the references to colour in this figure legend, the reader is referred to the web version of this article.)

image is obtained, but the "image" depends on pulse length and carrier envelope phase. Recall that in [4] the asymmetry was measured for $\mathrm{HCl}$ molecules using $50 \mathrm{fs}$ pulses (and circular polarized light), but this required the coincident detection of ions in order to restore the molecular-frame information. Based on a simplified tunneling model, the found asymmetry was attributed to the dipole moment and not to the orbital shape. Since a permanent or induced dipole moment influences the orientation-dependent ionization yield, this effect has to be corrected for, if orbital (densities) should be imaged. The size and importance of this correction increases with decreasing pulse length and thus, if a better time resolution should be obtained, by employing shorter pulses.

The orientation-dependent ionization of the HOMO-1 shows an asymmetry along the $z$ direction similar to the one discussed for the HOMO-2. Correspondingly, the mirror image (with respect to the $x y$ plane) is obtained for a phase $\phi=\pi$ and the asymmetry disappears for long pulses. Interestingly, a second asymmetry is observed when comparing the cuts through the $x z$ and $y z$ planes, shown on the absolute scale in Fig. 4. The lobes in the $x z$ plane are found to be substantially broader than the ones in the $y z$ plane. Since the discussed difference in the widths is perpendicular to the dipole moment (and shows the proper mirror symmetry), it is clearly a consequence of the shape of the orbital. In agreement with the orbital shape, the ion yield in the $x z$ plane is broader than in the $y z$ plane, since the orbital density of the HOMO- 1 is stretched in direction of the hydrogen atoms. However, for a sufficiently long pulse or an experiment with no control over the carrier envelope phase inversion symmetry is automatically enforced and thus the widths of the lobes pointing in positive or negative $z$ direction will be equal in such a case. The different width is thus only a consequence of the upper lobe (pointing toward $+z$ ) of the orbital in Fig. 4, while the lower lobe $(-z)$ dilutes the effect, since it is rotationally symmetric. 
This result is of interest, since it confirms directly that the orientation-dependent ionization reflects the position space orbital and not its momentum space counterpart, since position and momentum spaces are reciprocal and a broad distribution in position space corresponds to a narrow one in momentum space and vice versa. While this finding is in immediate agreement with the position-space based tunnel picture, it seems, on the first glance, to contradict the predictions of the strong-field approximation (SFA). Especially in its velocity-gauge version it predicts (within the frozen-core approximation) that the ionization rate of position-space orbital $\phi_{j}$ is related to its Fourier transform $\left\langle\mathbf{k}_{N} \mid \phi_{j}\right\rangle$ and thus to the momentum-space orbital [39],

$\Gamma_{j}^{(\mathrm{SFA})}=\sum_{N=N_{0}}^{\infty} \int \mathrm{d} \hat{\mathbf{k}}_{N} f\left(I, \omega, \hat{\boldsymbol{\epsilon}} \cdot \mathbf{k}_{N}\right) \quad\left|\left\langle\mathbf{k}_{N} \mid \phi_{j}\right\rangle\right|^{2}$.

The momentum $\mathbf{k}_{N}$ of the emitted electron depends on the number of absorbed photons $N$ that is limited below by the minimum number $N_{0}$ required to overcome the ionization threshold. The function $f$ depends on the laser intensity $I$ and frequency $\omega$ as well as on the projection of the momentum of the emitted electron on the laser polarization vector $\hat{\boldsymbol{\epsilon}}$. For sufficiently high laser intensities, $f$ peaks strongly at parallel emission of the electron [11] and the integral over all emission directions $\hat{\mathbf{k}}_{N}$ reduces (very) approximately to the integrand with $\hat{\mathbf{k}}_{N} \| \hat{\boldsymbol{\epsilon}}$. Furthermore, if the laser frequency is low and the intensity sufficiently high, but not (much) higher than the over-the-barrier ionization threshold, the function $f$ is strongly peaked at low momenta $\mathbf{k}_{N}$. Thus, according to SFA the lowmomentum part of the electron density is probed. Low momenta correspond, however, to the electrons far away from the nuclei, i.e. to the asymptotic part of the position-space orbital. Therefore, the predictions of the tunneling picture (probing the electron density close to the barrier) and the ones from the SFA (probing the low-momentum component of the momentum-space orbital) are compatible and also in agreement with the present findings for $\mathrm{H}_{2} \mathrm{O}$. Finally, in all formulations going beyond the frozen-core approximation, it is the Dyson orbital which should be more properly employed. However, the Dyson orbitals of $\mathrm{H}_{2} \mathrm{O}$ are predicted to resemble the usual orbitals, at least for the HOMO, HOMO- 1 , and HOMO-2 considered in this work [40]. Based on the tunneling picture of strong-field ionization, the interplay between position and momentum space representations of the orbital from which the electron is emitted is analyzed in more detail in [16] where a mixed representation (coordinate space along the field vector, momentum space perpendicular to it) is adopted.

Fig. 2 also shows the excitation probability of water for the given laser pulse. Again, the overall shape resembles the one of atomic $\mathrm{p}$ orbitals and is thus similar to the ionization yield. This similarity is most pronounced for the HOMO-1, while the HOMO excitation yield does not show the rotational symmetry around the $x$ axis, but is compressed in the $y$ direction. The HOMO-2 excitation yield shows on the other hand some additional structure that is absent in the ionization yield. The basic similarity of the orientational dependence of the excitation and ionization yields indicate the similarity of the processes leading to excitation and ionization as was discussed (for atomic helium) in [41]. A broad distribution of highly excited Rydberg states is populated, since (in the semi-classical picture) not all electron trajectories reach finally the continuum ("frustrated tunnel ionization").

The structures in some of the excitation spectra and their absence in the ionization yields indicate on the other hand the occurrence of resonances that do, however, barely influence the ionization process. An exception is the HOMO-1 (small) structure in the $y z$ plane that occurs close to the center. It is found in both the excitation and the ionization yields. Its origin becomes clearer from the comparison of the results that are obtained with the two
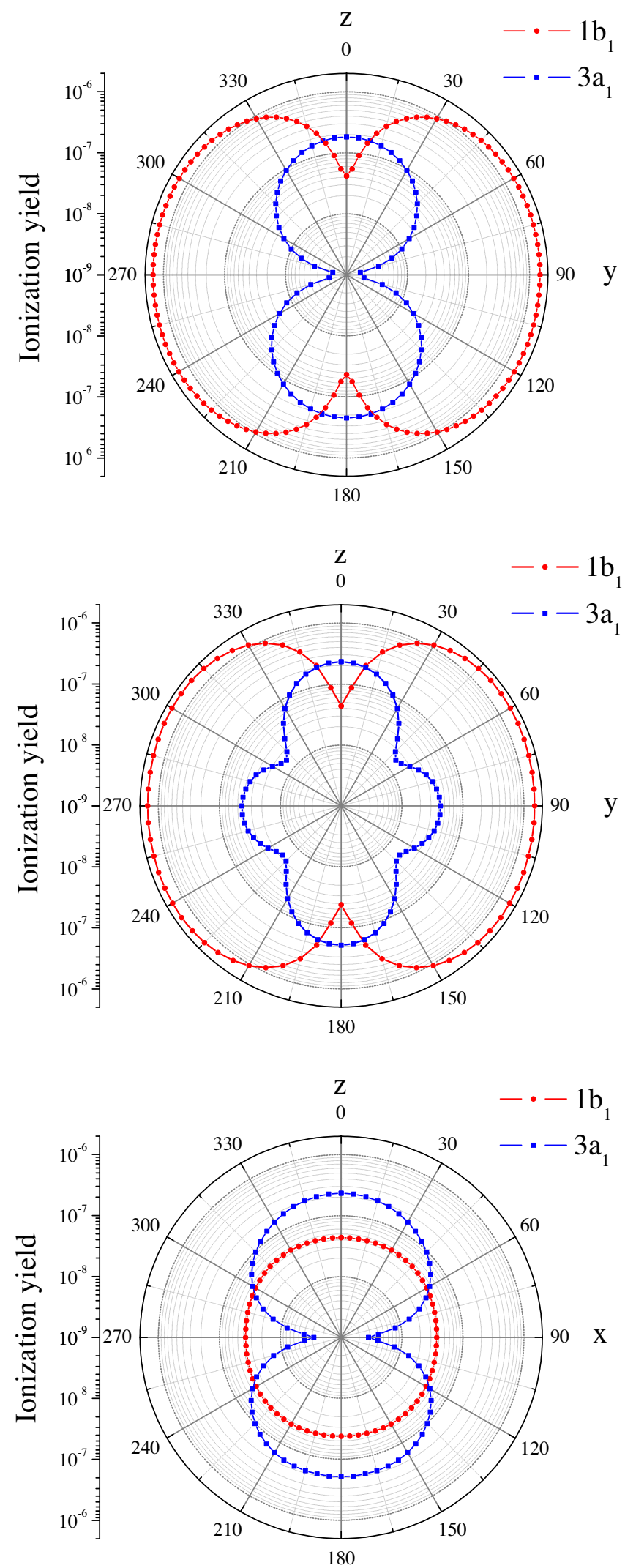

Fig. 5. Comparison of the respective absolute main ionization yields of the HOMO $1 b_{1}$ (red) and the HOMO- $13 a_{1}$ (blue) in the $y z$ plane (top and middle panels) and in the $x z$ plane (bottom panel). Laser parameters are the same as in Fig. $2.0^{\circ}$ mean alignment of the electric field polarization along the $z$ axis and $90^{\circ}$ along the $x$ or $y$ axis. The two upper graphs differ by the method applied: Method A (top) and method B (middle). (For interpretation of the references to colour in this figure legend, the reader is referred to the web version of this article.) 
different implementations of the SAE introduced in [13,32]. The results are shown in Fig. 5. Clearly, the structure occurs only in method $B$ in which all orbitals are propagated in time, but is absent in method $A$ in which all occupied field-free orbitals are excluded in the time propagation. In method A the ionization yields resemble in shape very well the one of atomic p orbitals. Note, also the small local minimum in the ionization yield of the HOMO that occurs along the $y$ axis is absent in method $A$. The reason for these structures seen in method $\mathrm{B}$ may be interpreted as a coupling between the HOMO and the HOMO-1. As the HOMO is partially depleted due to excitation and ionization, it can partially be refilled from the HOMO-1. Due to its lower binding energy, this transferred electron density is easier excited and ionized which leads to an enhanced excitation and ionization yield from the HOMO-1. At the same time, some population may be transferred from the HOMO into the HOMO-1 leading to a stabilization and thus a reduced excitation and ionization from the HOMO. A similar coupling of initially occupied (field-free) orbitals was found for $\mathrm{CO}_{2}$ [13], but there the influence on the ionization yield was much more pronounced. It should be understood, however, that this interpretation is given in terms of field-free eigenstates of the neutral molecule that are simply basis states in the TDSE calculation, and thus loose a direct physical meaning during the pulse. Furthermore, it may be reminded that within the spirit of the SAE approximation adopted here the transfer of population between orbitals occurs independently and is only driven by the laser field. Processes like correlated electron dynamics during tunneling as proposed in [42] are not considered.

For the visibility of the different orbitals in an experiment the absolute magnitude of the different ion yields is, of course, important. As is evident from Fig. 5, within the $x z$ plane the HOMO-1 ionization yield is much larger than the one from the HOMO due to the nodal plane of the latter. On the other hand, the space volume in which the HOMO-1 ionization yield dominates, is rather small, since it extends only to about $10 \%$ into the $y z$ plane. For the laser pulse adopted here, a very well aligned $\mathrm{H}_{2} \mathrm{O}$ sample would be required in order to observe HOMO-1 ionization. Integrated over all directions ionization is dominated by the one from the HOMO (note the logarithmic scale). A dominant ionization from the HOMO-1 in the $x z$ plane had been predicted in [23] based on a TDVFD calculation. A direct comparison (with identical 20 cycle pulses with peak intensity $5 \times 10^{13} \mathrm{~W} / \mathrm{cm}^{2}$ ) is shown in Fig. 6 .
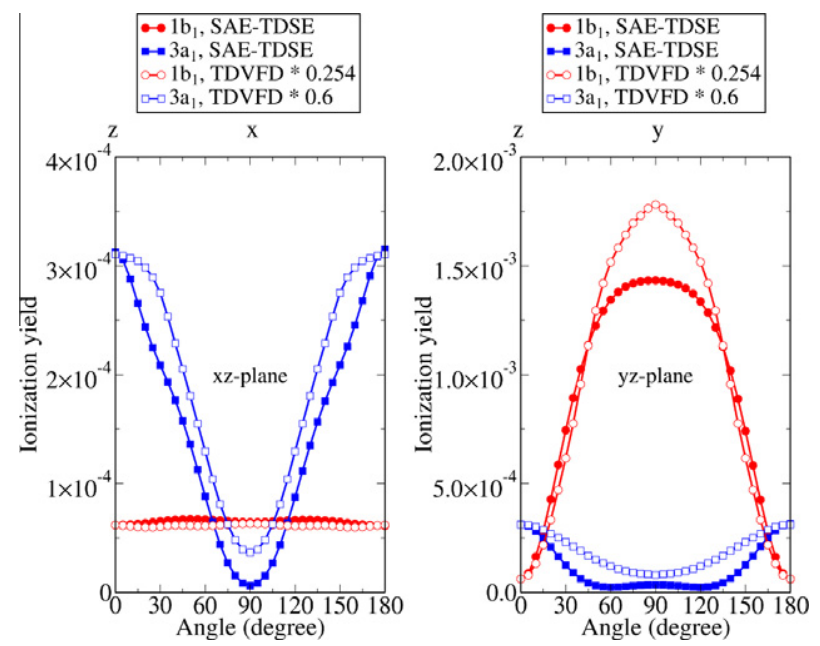

Fig. 6. Ionization yields in the $x z$ (left) and $y z$ plane (right) for the HOMO and HOMO- 1 for a $800 \mathrm{~nm}, 20$ cycles pulse at the intensity $5 \times 10^{13} \mathrm{~W} / \mathrm{cm}^{2}$. Our SAETDSE results are compared to TDVFD results [23]. The TDVFD data is scaled (by the factors given) to agree with the SAE-TDSE data at $0^{\circ}$. (For interpretation of the references to colour in this figure legend, the reader is referred to the web version of this article.)

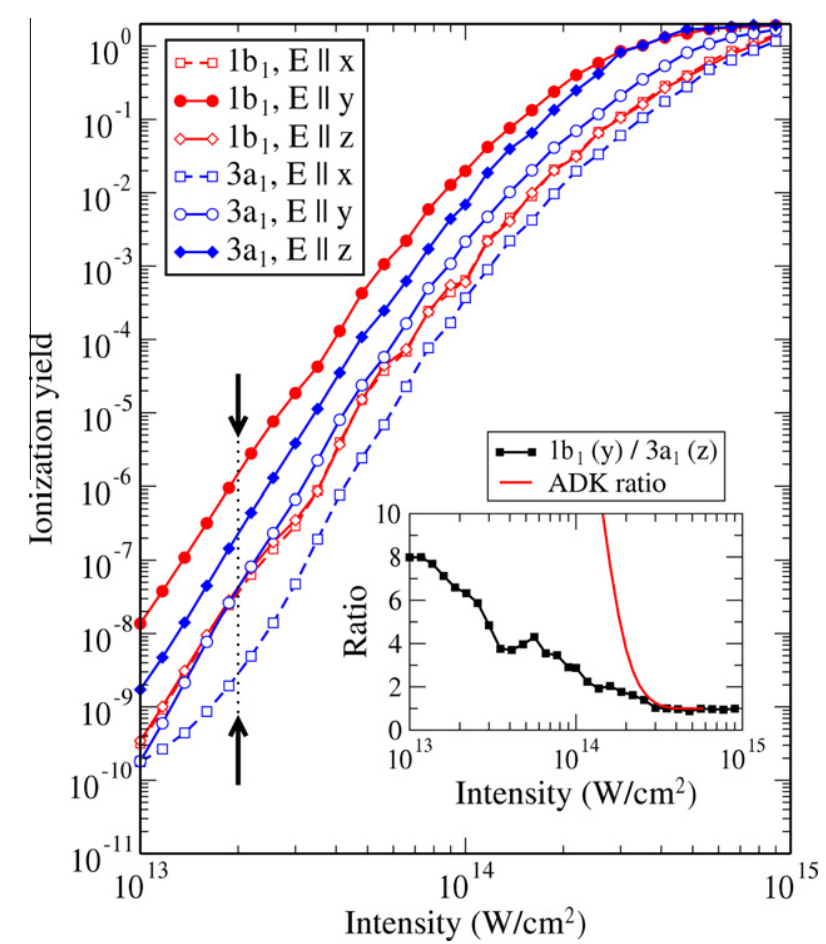

Fig. 7. Ionization yields of the HOMO $1 b_{1}$ (red) and the HOMO- $13 a_{1}$ (blue) for alignments of the electric field vector $\vec{E}$ along the three main axes $x$ (squares), $y$ (circles) and $z$ (diamonds) for 8 -cycle $\cos ^{2}$ laser pulses $(800 \mathrm{~nm})$. All ionization yields from the SAE-TDSE calculation were multiplied by a factor 2 . The inset shows the ratio of the HOMO ionized along the $y$-direction (red filled circles) and the HOMO-1 ionized along the $z$-direction (blue filled diamonds) together with an ADK estimate of the ratio. The arrows indicate the intensity used in most other graphs, especially in Fig. 2. (For interpretation of the references to colour in this figure legend, the reader is referred to the web version of this article.)

Qualitatively, the results are very similar, though the present ones tend to be broader, i.e. minima and maxima are slightly less pronounced. On the absolute scale, the results differ by factors in between 1.7 and 10 .

It is also interesting to consider the intensity dependence of the relative ionization yields of the HOMO and the HOMO-1 shown in Fig. 7. For low intensities, the maximum ionization occurs along the direction of the HOMO ( $y$ axis), followed by the one of the HOMO-1 along its maximum extension ( $z$ axis). The ionization of the HOMO along the $x$ and $z$ directions is practically identical and, for not too high intensities, agrees also to the one of the HOMO-1 along the $y$ direction. The lowest probability is found for the HOMO-1 along the $x$ direction, due to the absence of the resonant structure observed in $y$ direction and discussed above. Comparing the ionization probabilities of the HOMO and the HOMO-1 along the directions of maximum ionization (inset of Fig. 7) one finds a pronounced intensity dependence. Clearly, at high intensities the ratio approaches 1 , since the HOMO ionization saturates earlier than the one of the HOMO-1. In a comparison to experiment, saturation requires to consider also focal volume effects. Furthermore, the transition from a factor 2 to 1 due to saturation of the single-electron ionization should differ for HOMO and HOMO-1, if it follows the earlier findings for $\mathrm{H}_{2}$ where a rather sharp transition between factors 1 and 2 for about $10 \%$ ionization probability was found.

In a recent experiment the high-harmonic spectra of $\mathrm{H}_{2} \mathrm{O}$ and $\mathrm{D}_{2} \mathrm{O}$ have been measured. In the analysis based on a calculation using the present theoretical approach it was concluded that the harmonics for the different isotopes differ significantly, because ionization from the HOMO-1 excites a vibrational wavepacket, 
while HOMO ionization does not [6]. In view of the present finding it is clear that the observability of the HOMO-1 high harmonics in an isotropic sample requires that the lower ionization probability is partially compensated for by the higher recombination rate (3rd step of the three-step model). Furthermore, as is also seen from the present results, saturation plays an important role for higher intensities, since the ratio of the maxima of the ionization yields from the HOMO and the HOMO-1 approaches 1 for high intensities (cf. the inset of Fig. 7). An estimate of this ratio with ADK (including the saturation effect) is also shown. Clearly, ADK overestimates the ratio significantly for lower intensities and reaches the ratio 1 due to saturation very abruptly. Concerning the direct imaging scheme discussed above, the intensity dependence of the ratio is important, since the image of the HOMO density may be distorted by an admixture of lower lying orbitals and this admixture changes with intensity. While this is very critical for a measurement at a single intensity, the intensity dependence of the ratio can, on the other hand, also help to disentangle the contributions from different orbitals.

\section{Conclusion}

In conclusion, the response of water molecules to ultrashort intense laser pulses (at $800 \mathrm{~nm}$ ) was investigated by solving the time-dependent Schrödinger equation within a single-active-electron approximation, but taking into account a complete multicenter molecular core. In fact, the influence of the laser field on all electrons was considered, but the electron-electron interaction is restricted to a single-determinant approximation. It was found that the orientation-dependent ionization from the three energetically highest occupied orbitals reflects their overall shape. However, for a sufficiently long pulse the image acquires inversion symmetry, since the electric field vector points approximately symmetrically up and down. This enforced inversion symmetry can be prevented, if the emission of the electrons is angularly resolved or, at least, electrons measured in the up- or downward direction with respect to the molecular orientation are discriminated. Alternatively, the angle-resolved ion distribution (after dissociation) as in [4] could be used to obtain molecular-frame resolved information. For short pulses, in the present case even as long as 8 cycles, deviations from the inversion symmetry of the ionization distribution were found. Although it may be a consequence of the asymmetric shape of the orbital, the visibility of this effect even for such relatively long pulses suggests that it is mainly due to the permanent dipole moment of an (oriented) water molecule. While this effect provides additional information, it distorts the image of the orbitals and it depends evidently on the absolute phase and time duration of the laser pulse. On the other hand, the missing rotational symmetry of water compared to the previously mainly discussed linear molecules allowed a distinction of broad and narrow features of the orbitals, provided they survive the enforced inversion symmetry.

The authors thank S.-K. Son for kindly providing their data in numerical form, A. Hussien for the ADK data, and COST CM0702 for financial support. SP, AS, and PD acknowledge financial support within the EU Initial Training Network (ITN) CORINF, SP and AS from the Fonds der Chemischen Industrie, AC from the Spanish MIC-
INN (grant FIS2009-13364-C02-01), and PD by CNR-INFM Democritos and INSTM Crimson.

\section{References}

[1] J. Itatani, J. Levesque, D. Zeidler, H. Niikura, H. Pépin, J.C. Kieffer, P.B. Corkum, D.M. Villeneuve, Nature 432 (2004) 867.

[2] M. Meckel, D. Comtois, D. Zeidler, A. Staudte, D. Pavičić, H.C. Bandulet, H. Pépin, J.C. Kieffer, R. Dörner, D.M. Villeneuve, P.B. Corkum, Science 320 (2008) 1478.

[3] B.K. McFarland, J.P. Farrell, P.H. Bucksbaum, M. Gühr, Science 322 (2008) 1232

[4] H. Akagi, T. Otobe, A. Staudte, A. Shiner, F. Turner, R. Dorner, D.M. Villeneuve, P.B. Corkum, Science 325 (2009) 1364.

[5] O. Smirnova, Y. Mairesse, S. Patchkovskii, N. Dudovich, D. Villeneuve, P. Corkum, M.Y. Ivanov, Nature 460 (2009) 972.

[6] J.P. Farrell, S. Petretti, J. Förster, B.K. McFarland, L.S. Spector, Y.V. Vanne, P. Decleva, P.H. Bucksbaum, A. Saenz, M. Gühr, Phys. Rev. Lett. 107 (2011) 083001.

[7] M. Lein, J. Phys. B 40 (2007) R135.

[8] C.D. Lin, A.-T. Le, Z. Chen, T. Morishita, R. Lucchese, J. Phys. B 43 (2010) 122001

[9] E. Goll, G. Wunner, A. Saenz, Phys. Rev. Lett. 97 (2006) 103003.

[10] T. Ergler, B. Feuerstein, A. Rudenko, K. Zrost, C.D. Schröter, R. Moshammer, J. Ullrich, Phys. Rev. Lett. 97 (2006) 103004.

[11] Y.V. Vanne, A. Saenz, Phys. Rev. A 82 (2010) 011403.

[12] D. Pavičić, K.F. Lee, D.M. Rayner, P.B. Corkum, D.M. Villeneuve, Phys. Rev. Lett. 98 (2007) 243001

[13] S. Petretti, Y.V. Vanne, A. Saenz, A. Castro, P. Decleva, Phys. Rev. Lett. 104 (2010) 223001.

[14] I. Thomann, R. Lock, V. Sharma, E. Gagnon, S.T. Pratt, H.C. Kapteyn, M.M. Murnane, W. Li, J. Phys. Chem. A 112 (2008) 9382.

[15] M. Abu-samha, L.B. Madsen, Phys. Rev. A 80 (2009) 023401.

[16] R. Murray, M. Spanner, S. Patchkovskii, M.Y. Ivanov, Phys. Rev. Lett. 106 (2011) 173001.

[17] S. Baker, J.S. Robinson, C.A. Haworth, H. Teng, R.A. Smith, C.C. Chirilă, M. Lein, J.W.G. Tisch, J.P. Marangos, Science 312 (2006) 424.

[18] M. Yamazaki, J. Adachi, T. Teramoto, A. Yagishita, M. Stener, P. Decleva, J. Phys. B 42 (2009) 051001

[19] A. Staudte, S. Patchkovskii, D. Pavičić, H. Akagi, O. Smirnova, D. Zeidler, M. Meckel, D.M. Villeneuve, R. Dörner, M.Y. Ivanov, P.B. Corkum, Phys. Rev. Lett. 102 (2009) 033004.

[20] Y.-J. Jin, X.-M. Tong, N. Toshima, Phys. Rev. A 83 (2011) 063409.

[21] P. Krause, T. Klamroth, P. Saalfrank, J. Chem. Phys. 127 (2007) 034107.

[22] S. Borbély, K. Tőkési, L. Nagy, Eur. Phys. J. D 59 (2010) 337.

[23] S.-K. Son, S.-I. Chu, Chem. Phys. 366 (2009) 91.

[24] S.-F. Zhao, J. Xu, C. Jin, A.-T. Le, C.D. Lin, J. Phys. B 44 (2011) 035601.

[25] M.C.H. Wong, J.-P. Brichta, V.R. Bhardwaj, Opt. Lett. 35 (2010) 1947.

[26] S.-F. Zhao, C. Jin, R.R. Lucchese, A.-T. Le, C.D. Lin, Phys. Rev. A 83 (2011) 033409.

[27] M. Falge, V. Engel, M. Lein, Phys. Rev. A 81 (2010) 023412.

[28] A. Saenz, J. Phys. B 33 (2000) 4365.

[29] X. Urbain, B. Fabre, E.M. Staicu-Casagrande, N. de Ruette, V.M. Andrianarijaona, J. Jureta, J.H. Posthumus, A. Saenz, E. Baldit, C. Cornaggia, Phys. Rev. Lett. 92 (2004) 163004.

[30] S. Patchkovskii, Phys. Rev. Lett. 102 (2009) 253602.

[31] M. Awasthi, Y.V. Vanne, A. Saenz, A. Castro, P. Decleva, Phys. Rev. A 77 (2008) 063403.

[32] S. Petretti, A. Saenz, A. Castro, P. Decleva, in preparation.

[33] R. van Leeuwen, E.J. Baerends, Phys. Rev. A 49 (1994) 2421

[34] D. Toffoli, M. Stener, G. Fronzoni, P. Decleva, Chem. Phys. 276 (2002) 25.

[35] M. Spanner, S. Patchkovskii, Phys. Rev. A 80 (2009) 063411.

[36] M.S. Banna, D.A. Shirley, J. Chem. Phys. 63 (1975) 4759.

[37] G. Herzberg, Molecular Spectra and Molecular Structure III: Electronic spectra and electronic structure of polyatomic molecules, Van Nostrand, New York, 1966.

[38] S. Hamel, P. Duffy, M.E. Casida, D.R. Salahub, J. Electr. Spectros. Relat. Phenom. 123 (2002) 345.

[39] A. Becker, F.H.M. Faisal, J. Phys. B 38 (2005) R1.

[40] G.M. Seabra, I.G. Kaplan, V.G. Zakrewski, J.V. Ortiz, J. Chem. Phys. 121 (2004) 4143.

[41] T. Nubbemeyer, K. Gorling, A. Saenz, U. Eichmann, W. Sandner, Phys. Rev. Lett. 101 (2008) 233001

[42] Z.B. Walters, O. Smirnova, Attosecond correlation dynamics during electron tunnelling from molecules, J. Phys. B 43 (2010) 161002. 